\title{
Correction to: The disengagement of visual attention in the gap paradigm across adolescence
}

\author{
S. Van der Stigchel ${ }^{1} \cdot$ R. S. Hessels ${ }^{2} \cdot$ J. C. van Elst ${ }^{2} \cdot$ C. Kemner ${ }^{2,3}$
}

Published online: 6 July 2019

○) Springer-Verlag GmbH Germany, part of Springer Nature 2019

Correction to: Exp Brain Res (2017) 235:3585-3592

https://doi.org/10.1007/s00221-017-5085-2

In the manuscript, we write that saccadic reaction time was determined as the time between the offset of the last fixation on the central stimulus and the onset of the peripheral stimulus (the target). This is incorrect. We determined saccadic reaction time as the time between the onset of the first fixation on the target and the onset of the target. Trials with saccadic reaction times shorter than $80 \mathrm{~ms}$ were excluded from the analyses. Importantly, the conclusions are the same irrespective of the calculation.

The original article can be found online at https://doi.org/10.1007/ s00221-017-5085-2.

S. Van der Stigchel

S.VanderStigchel@uu.nl

1 Department of Experimental Psychology, Helmholtz Institute, Utrecht University, Heidelberglaan 1, 3584 CS Utrecht, The Netherlands

2 Developmental Psychology, Utrecht University, Utrecht, The Netherlands

3 Brain Centre Rudolf Magnus, University Medical Center, Utrecht, The Netherlands 